\title{
Dark-Matter-Induced Violation of the Weak Equivalence Principle
}

\author{
Sean M. Carroll, ${ }^{1}$ Sonny Mantry, ${ }^{1}$ Michael J. Ramsey-Musolf, ${ }^{2,1}$ and Christoper W. Stubbs ${ }^{3}$ \\ ${ }^{1}$ California Institute of Technology, Pasadena, California 91125, USA \\ ${ }^{2}$ University of Wisconsin-Madison, Madison, Wisconsin 53706, USA \\ ${ }^{3}$ Harvard-Smithsonian Center for Astrophysics, Cambridge, Massachusetts 02138, USA \\ (Received 30 July 2008; revised manuscript received 15 October 2008; published 1 July 2009)
}

\begin{abstract}
A long-range fifth force coupled to dark matter can induce a coupling to ordinary matter if the dark matter interacts with standard model fields. We consider constraints on such a scenario from both astrophysical observations and laboratory experiments. We also examine the case where the dark matter is a weakly interacting massive particle, and derive relations between the coupling to dark matter and the coupling to ordinary matter for different models. Currently, this scenario is most tightly constrained by galactic dynamics, but improvements in Eötvös experiments can probe unconstrained regions of parameter space.
\end{abstract}

A light scalar field $\phi$ coupled to dark matter (DM) could mediate a long-range force of strength comparable to gravity. A number of models along these lines have been proposed, motivated both by attempts to account for features in the distribution of DM and to explore interactions with quintessence [1-12]. Interesting limits on such a force have been derived from observations of DM dynamics in the tidal stream of the Sagittarius dwarf galaxy $[13,14]$. The detection of a new long-range force would signal the presence of a new mass hierarchy, between the light scalar mass $m_{\phi}<10^{-25} \mathrm{eV}$ and the weak scale $m_{W} \sim 100 \mathrm{GeV}$. Thus, if new long-range forces exist in the dark sector, their observation could provide a new window on other puzzling scale hierarchies in particle physics, such as that between $m_{W}$ and the Planck scale, $M_{P} \sim 10^{19} \mathrm{GeV}$.

If the scalar couples to standard model (SM) fields, it will give rise to a composition-dependent force acting on ordinary matter [15]; such forces are tightly constrained by Eötvös experiments looking for violations of the Weak Equivalence Principle (WEP) [16]. On the other hand, even if $\phi$ has only an elementary (i.e., renormalizable) coupling to DM, interactions between DM and ordinary matter will still induce a coupling of $\phi$ to ordinary matter. This can be thought of as arising from the scalar coupling to virtual DM particles in ordinary atomic nuclei. We therefore naturally expect a fifth force coupled to the SM if a light scalar couples to a DM field having SM interactions. In what follows, we show how this scenario may arise in simple model illustrations and analyze modeldependent details of its viability in a subsequent publication. (As this Letter was being prepared for submission, we became aware of closely related work by Bovy and Farrar [17]. For a detailed comparison see [18]).

This Letter has two goals. First, we consider the varieties of experimental constraints on a two-dimensional parameter space, given by the respective couplings of a new longrange force to ordinary matter and to dark matter. Eötvös experiments are sensitive to anomalous accelerations of ordinary matter toward the DM in the galactic center; however, we argue on general grounds that any force capable of giving rise to a detectable effect would first give rise to a detectable fifth force acting between two sources of ordinary matter. Our second goal is to explore illustrative scenarios in which a light scalar couples at tree level only to a weakly interacting massive particle (WIMP) DM candidate, and derive the induced coupling to ordinary matter.

Fifth-force phenomenology.-We now turn to order-ofmagnitude estimates of the available experimental constraints on couplings of a light scalar $\phi$ to both ordinary matter and to DM. We assume the existence of a mechanism that keeps its mass small. The static potential between a test object $o$ and a source $s$ arising from the combined effects of gravity and the coupling to $\phi$ is then

$$
V=-\frac{G M_{o} M_{s}}{r}\left(1+\frac{1}{4 \pi G} \frac{q_{o} q_{s}}{\mu_{o} \mu_{s}}\right),
$$

where $q / \mu$ is the charge per unit mass and $G=M_{P}^{-2}$; for a fermion $\psi_{i}$ with mass $m_{i}$ and Yukawa coupling $\mathcal{L}=$ $g_{i} \phi \bar{\psi}_{i} \psi_{i}$, we have $q / \mu=g_{i} / m_{i}$. Searches for WEPviolating fifth forces place limits on the Eötvös parameter,

$$
\eta=2 \frac{\left|a_{1}-a_{2}\right|}{\left|a_{1}+a_{2}\right|},
$$

where $a_{1}$ and $a_{2}$ are the accelerations of two bodies with different compositions.

We will assume that the dominant couplings of $\phi$ to ordinary matter are to protons $(p)$, neutrons $(n)$, and electrons $(e)$, neglecting, for example, couplings to atomic binding energy. (See, e.g., [19-21].) Then, for a neutral object made of ordinary matter, we can define

$$
\frac{g_{p}+g_{e}}{m_{p}+m_{e}} \equiv \frac{g_{*}}{m_{p}}, \quad \frac{g_{n}}{m_{n}} \equiv(1+\epsilon) \frac{g_{*}}{m_{p}} .
$$

The parameter $\epsilon$ can be calculated by first computing the 
coupling of $\phi$ to nucleons by matching onto its coupling to quarks, $g_{N}=\sum g_{i}\left\langle N\left|\bar{q}_{i} q_{i}\right| N\right\rangle$, where the sum is over all quarks. We also assume that $g_{i}$ for quarks and leptons is proportional to the mass of the fermion, so we can write $g_{i}=\bar{g} m_{i} / m_{p}$; this assumption holds true in simple models, as we show below. We obtain $g_{N}$ by comparing the energy momentum tensor in six-flavor and three-flavor QCD [22] and using the known light-quark nucleon matrix elements [23]. We find $g_{p} \sim 0.4 \bar{g} \approx g_{n}$. For the electron we have $g_{e}=\bar{g} m_{e} / m_{p}$, which leaves us with $\epsilon=-8 \times$ $10^{-4}$.

Assuming that the fifth force is much weaker than gravity, the Eötvös parameter with both test bodies and source constructed from ordinary matter is then

$$
\eta_{\mathrm{OM}} \approx \frac{M_{P}^{2}}{4 \pi}\left|\epsilon\left(f_{1}-f_{2}\right)\right|\left(\frac{g_{*}}{m_{p}}\right)^{2},
$$

where $f_{i}=Z_{i} / A_{i}$ is the nuclear fraction of protons in body $i$, and "OM" denotes a source consisting of ordinary matter. Atomic binding energy corrections may introduce $\mathcal{O}(1)$ corrections to this relation but do not substantially affect our conclusions [19]. The current best limit on $\eta$ from torsion-balance experiments involving terrestrial test bodies is $\eta_{\mathrm{OM}}<2 \times 10^{-13}$, using materials with $f_{1}-$ $f_{2} \sim 2 \times 10^{-2}$ [16]. From this, we derive $g_{*} / m_{p}<3 \times$ $10^{-23} \mathrm{GeV}^{-1}$, which we have plotted as a vertical line in Fig. 1.

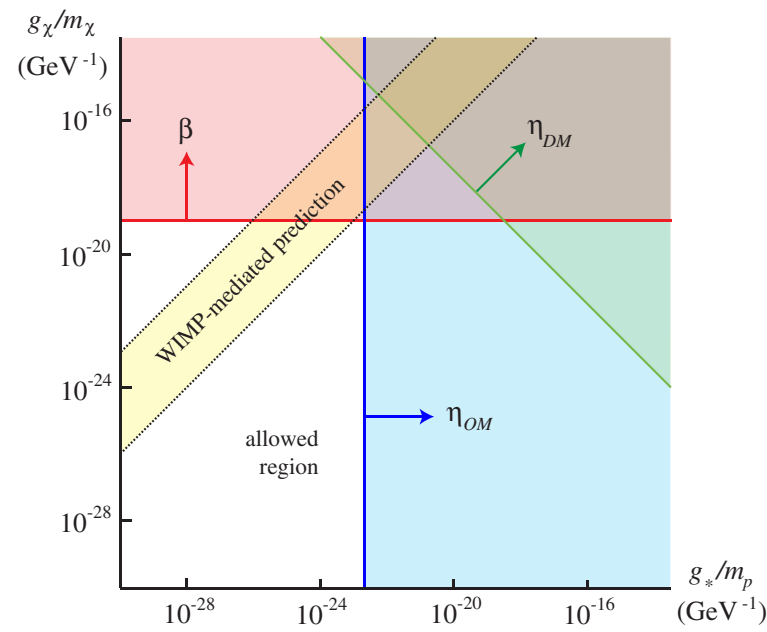

FIG. 1 (color online). Constraints on the strength of fifth forces coupled to ordinary matter and/or dark matter. The horizontal axis is the effective coupling to ordinary matter $g_{*}$ divided by the proton mass $m_{p}$, while the vertical axis is the Yukawa coupling to DM $g_{\chi}$ divided by the mass $m_{\chi}$ of the DM, both in units of inverse GeV. The vertical line (in blue) is the constraint from Eötvös experiments with ordinary-matter sources; the horizontal line (in red) is from DM tidal tails; and the diagonal line in the top right quadrant (in green) is from the searches for anomalous accelerations in the direction of the Galactic center. The diagonal band running from bottom left to top right (in yellow) is the range of predictions from the WIMP models we consider.
Now consider a fermionic dark matter particle $\chi$ coupled via $\mathcal{L}=g_{\chi} \phi \bar{\chi} \chi$. Interesting limits may be obtained on anomalous accelerations of laboratory test bodies in the direction of the galactic center, where the dominant source is presumably the DM inside the Solar circle [24]. For a source made of DM and test bodies of ordinary matter, we obtain

$$
\eta_{\mathrm{DM}} \approx \frac{M_{P}^{2}}{4 \pi}\left|\epsilon\left(f_{1}-f_{2}\right)\right| \frac{g_{*} g_{\chi}}{m_{p} m_{\chi}},
$$

where "DM" denotes accelerations sourced by dark matter. The best current limits on anomalous accelerations in the direction of the galactic center give $\eta_{\mathrm{DM}}<10^{-5}$ [16], corresponding to $g_{*} g_{\chi} / m_{p} m_{\chi}<5 \times 10^{-38} \mathrm{GeV}^{-2}$. This is plotted as a downward-sloping diagonal line in Fig. 1.

Separate limits on $g_{\chi} / m_{\chi}$ are obtained from astrophysical tests, such as the dynamics of galactic tidal streams $[13,14]$. In this case, the constraint limits the strength of the force due to $\phi$ relative to that due to gravity, rather than a composition-dependent acceleration. The relevant parameter is

$$
\beta=\frac{M_{P}}{\sqrt{4 \pi}} \frac{g_{\chi}}{m_{\chi}} .
$$

For reasonable models of the Sagittarius tidal stream, we obtain $\beta<0.2[13,14]$, corresponding to $g_{\chi} / m_{\chi}<$ $10^{-19} \mathrm{GeV}^{-1}$, plotted as a horizontal line in Fig. 1 .

From Fig. 1, it is clear that current bounds from constraints on anomalous accelerations toward the galactic center do not cover any region of parameter space that is not already excluded by constraints on the couplings to ordinary-matter and DM alone. Assuming that limits on $\beta$ do not appreciably improve, $\eta_{\text {DM }}$ will only probe unconstrained parameter space once it is more sensitive to $g_{*} / m_{p}$ for $g_{\chi} / m_{\chi} \sim 10^{-19} \mathrm{GeV}^{-1}$ (the value along the $\beta$ constraint line) than $\eta_{\mathrm{OM}}$. Although any improvement in sensitivity to $\left|a_{1}-a_{2}\right|$ will lead to the same improvements in $\eta_{\mathrm{DM}}$ and $\eta_{\mathrm{OM}}$, they depend linearly and quadratically on $g_{*} / m_{p}$, respectively. Since the $\eta_{\mathrm{DM}}$ constraint currently is weaker by a factor of $10^{4}$ than the $\eta_{\mathrm{OM}}$ bound along the $\beta$ constraint line, one must improve sensitivity to $\left|a_{1}-a_{2}\right|$ by at least $\sim 10^{8}$. This corresponds to $\eta_{\mathrm{OM}} \sim 10^{-21}$, $\eta_{\mathrm{DM}} \sim 10^{-13}, \quad g_{*} / m_{p} \sim 10^{-27} \mathrm{GeV}^{-1}$. The proposed STEP experiment aims at $\eta_{\mathrm{OM}}<10^{-17}$ [25], not enough to achieve this goal. If an anomalous acceleration toward the galactic center were detected with $\eta_{\mathrm{DM}}>10^{-13}$ but with no corresponding detection of $\eta_{\mathrm{OM}}$, it could not be accommodated by the type of theory considered here.

Model examples: WIMP dark matter-The most popular DM models involve WIMPs-stable neutral particles $\chi$ living in some representation of the electroweak gauge and Poincaré groups. Their interactions with electroweak gauge bosons provide an annihilation cross section that leads to cosmologically interesting relic abundances. A classification of the various possible representations containing a viable DM candidate can be found in [26]. Here, 


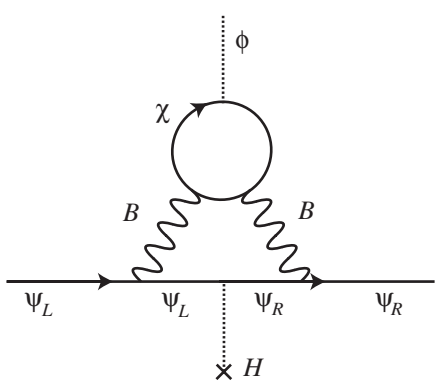

FIG. 2. Two-loop graph inducing an interaction between a massless scalar $\phi$ and a Dirac fermion $\psi$, mediated by a WIMP $\chi$ and hypercharge gauge bosons $B$.

we select a few cases to illustrate the range of scenarios for WIMP-induced fifth-force couplings to ordinary matter.

Figures 2 and 3 show the lowest-order processes that generate the gauge-invariant interaction

$$
c_{i} \phi \bar{\psi}_{i L} H \psi_{i R} / \Lambda+\text { H.c. }
$$

involving $\phi$, the Higgs doublet $H$, and the left-handed doublet and right-handed singlet components of a SM fermion $\psi_{i}$. Here, $\Lambda$ is a mass scale associated with the particles in the loops and $c_{i}$ is proportional to the fermion Yukawa coupling $y_{i}$. After electroweak symmetry breaking, in which the neutral component of $H$ obtains a vacuum expectation value $v=246 \mathrm{GeV}$, this interaction yields the coupling $g_{i} \phi \bar{\psi}_{i} \psi_{i}$ with $g_{i} \sim m_{i} / \Lambda$.

Since right-handed fermions do not couple to the $\mathrm{SU}(2)_{L}$ gauge bosons $W^{a}$, Fig. 2 only contributes if the WIMP $\chi$ has nonvanishing hypercharge. In that case, the hypercharge gauge boson $B$ can couple $\chi$ to both $\psi_{i L}$ and $\psi_{i R}$. The "Compton scattering" process of Fig. 3, in contrast, contributes for all WIMPs, with those having $Y=0$ receiving contributions only from internal $\mathrm{SU}(2)_{L}$ gauge bosons. The two-loop subgraph of Fig. 3 generates the structure $\not p+\not P^{\prime}$ involving the incoming and outgoing fermion momenta. The $\not p^{\prime}$ cancels the $1 / \not p^{\prime}$ of the intermediate $\psi_{L}$, leading to the momentum-independent interaction (7). Formally, the two-loop subgraph of Fig. 3along with diagrams involving external gauge boson insertions (not shown)-yields the operator $\bar{\psi}_{L} i(\overleftarrow{\not D}-\overrightarrow{\not D}) \psi_{L}$

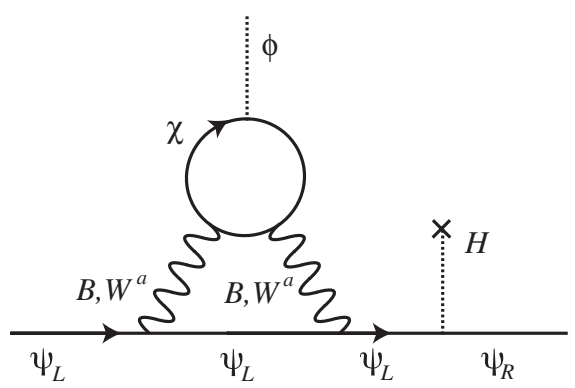

FIG. 3. Two-loop "Compton scattering" graph inducing an interaction between a massless scalar $\phi$ and a Dirac fermion $\psi$, mediated by a WIMP $\chi$ and electroweak gauge bosons. that is equivalent to (7) by virtue of the equation of motion for $\psi_{L}$.

The simplest realization of this scenario occurs when $\chi$ is a scalar $\mathrm{SU}(2)_{L}$ doublet, having an elementary $g_{\chi} \phi \chi^{\dagger} \chi$ interaction with the long-range force mediator. A mass $m_{\chi} \sim 0.5 \mathrm{TeV}$ is needed to obtain the observed DM relic density [26]. A fermionic realization requires the presence of two doublets with $Y= \pm 1$ to cancel anomalies and $m_{\chi}$ of order $1 \mathrm{TeV}$. For either case, the graph in Fig. 2 is finite and a simple estimate yields

$$
g_{i} \sim\left(\frac{\alpha_{\mathrm{em}}}{4 \pi}\right)^{2} \frac{m_{i}}{m_{\chi}} g_{\chi}
$$

The contribution from Fig. 3 is naïvely an order of magnitude larger since it involves four powers of the $\mathrm{SU}(2)_{L}$ gauge coupling that is roughly twice as large as the $\mathrm{U}(1)_{Y}$ coupling entering Fig. 2:

$$
g_{i} \sim\left(\frac{\alpha_{\mathrm{em}}}{\pi}\right)^{2} \frac{m_{i}}{m_{\chi}} g_{\chi}
$$

In terms of the nucleon coupling $g_{*}$ defined in (3), the estimate (8) becomes

$$
\frac{g_{*}}{m_{p}} \sim 10^{-7} \frac{g_{\chi}}{m_{\chi}} .
$$

This provides a lower limit (upper dotted line in Fig. 1) on the coupling strength induced by WIMP dark matter, and would be relevant if $\chi$ were a singlet of $\mathrm{SU}(2)_{L}$ with nonzero hypercharge. If $\chi$ is a doublet or triplet of $\mathrm{SU}(2)_{L}$, Eq. (9) applies, and the resulting coupling is an order of magnitude larger, $g_{*} / m_{p} \sim 10^{-6} g_{\chi} / m_{\chi}$.

Realistic implementations of the WIMP idea often introduce more than just a single DM field (and its charged partners). In supersymmetry, for example, the $\chi$ is the lightest supersymmetric particle (LSP) and is a linear superposition of the superpartners of the electroweak gauge bosons (winos and binos) and Higgs bosons (Higgsinos). In addition, one has scalar squarks and sleptons $\tilde{\psi}$ that interact with their partners and the LSP via an interaction $\lambda \tilde{\psi} \bar{\psi} \chi+$ H.c. If $\phi$ is the scalar component of a singlet superfield $\hat{S}$, a superpotential term of the form $\hat{S} \hat{H}_{u} \cdot \hat{H}_{d}$ will generate a coupling of $\phi$ to the higgsino components of the LSP. As a result, we expect a one-loop coupling of $\phi$ to SM fermions, as shown in Fig. 4, which gives

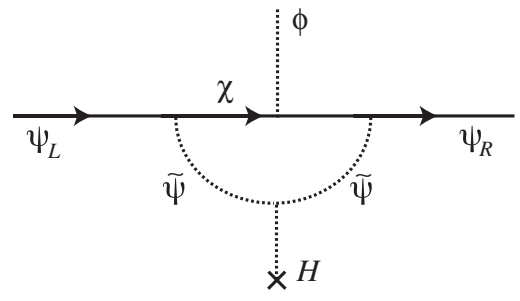

FIG. 4. One-loop graph involving scalar sfermions $\tilde{\psi}$. 


$$
g_{i} \sim \frac{1}{16 \pi^{2}} \frac{m_{i} \mu \lambda^{2}}{M_{\text {susy }}^{2}} g_{\chi},
$$

where $\mu$ is the $\mu$ parameter of supersymmetry and $M_{\text {susy }}$ is the mass of the heaviest superparticle in the loop. We assume for simplicity that $m_{\chi} \approx M_{\text {susy }} \approx \mu \approx v$. The most favorable case is when the DM $\chi$ is primarily a bino, in which case $\lambda=g_{1}$, the $\mathrm{U}(1)_{Y}$ gauge coupling. This implies

$$
\frac{g_{*}}{m_{p}} \sim 10^{-4} \frac{g_{\chi}}{m_{\chi}}
$$

leading to the lower dotted line in Fig. 1. In this case, where the existence of the squarks enables a one-loop contribution, the coupling to ordinary matter is enhanced by a factor of order $10^{3}$. This is the most optimistic scenario, in the sense of creating the strongest coupling of $\phi$ to ordinary matter. If $\chi$ is predominantly higgsino, the coupling $\lambda$ will be approximately the Higgs Yukawa coupling to $\psi_{i}$, which is order one for the top quark. Figure 1 plots the range of values in different models, from a minimal WIMP scenario that only couples $\phi$ to ordinary matter at two loops, to a binolike model (with sfermions) that implies a contribution at one loop. Higgsino-like DM, to be considered in future work, could live outside the WIMP band in Fig. 1 due to a Yukawa enhanced coupling to the top quark. For $\phi$ coupling to ordinary matter by mixing with the Higgs, see [18]. Similarly, in any given model of nonsterile DM coupled to a long-range force, one can determine the size of the induced coupling to ordinary matter and its implications for Eötvös experiments.

Conclusions.-We have found that a weakly interacting dark matter particle $\chi$ coupled to a light scalar $\phi$ with strength $g_{\chi}$ naturally induces an effective coupling to ordinary matter with strength $g_{*} / m_{p} \sim\left(10^{-7}-\right.$ $\left.10^{-4}\right) g_{\chi} / m_{\chi}$. The low end of this range corresponds to minimal WIMP models with only higher-loop contributions to the interaction of $\phi$ with SM fields, through $\mathrm{SU}(2)_{L}$ gauge bosons, while the high end corresponds to binolike DM with one-loop contributions. Comparing with Fig. 1, we see that the best current limits on these models come from purely astrophysical bounds on new long-range forces in the dark sector; if improvements in these techniques discovered such a force, it would predict a new force between ordinary matter if the DM were WIMPs. Meanwhile, improvements in the sensitivity of Eötvös-type experiments could provide interesting new constraints on a WIMP-mediated coupling. Any improvement of the limits on $g_{*} / m_{p}$ would begin cutting into the predictions of the models examined here (at the order-of-magnitude precision we considered). Currently, constraints on a fifth force in the direction of the galactic center do not independently constrain any of the parameter space; measurements of anomalous accelerations would have to improve by a factor of about $10^{8}$ before they would begin to do so.
We thank J. Distler, M. Kamionkowski, T. Tait, M. Wise, and K. Zurek for helpful conversations. This research was supported in part by Department of Energy Contracts No. DE-FG03-92-ER40701 and No. DE-FG0208ER41531, the Wisconsin Alumni Research Foundation, and the Gordon and Betty Moore Foundation.

[1] T. Damour, G. W. Gibbons, and C. Gundlach, Phys. Rev. Lett. 64, 123 (1990).

[2] J.A. Frieman and B.-A. Gradwohl, Phys. Rev. Lett. 67, 2926 (1991).

[3] B.-A. Gradwohl and J. A. Frieman, Astrophys. J. 398, 407 (1992).

[4] G. W. Anderson and S.M. Carroll, arXiv:astro-ph/ 9711288.

[5] S. M. Carroll, Phys. Rev. Lett. 81, 3067 (1998).

[6] L. Amendola and D. Tocchini-Valentini, Phys. Rev. D 66, 043528 (2002).

[7] G. R. Farrar and P. J.E. Peebles, Astrophys. J. 604, 1 (2004).

[8] S. S. Gubser and P. J. E. Peebles, Phys. Rev. D 70, 123511 (2004).

[9] S. S. Gubser and P. J. E. Peebles, Phys. Rev. D 70, 123510 (2004).

[10] O. Bertolami and J. Paramos, Phys. Rev. D 71, 023521 (2005).

[11] A. Nusser, S. S. Gubser, and P. J. E. Peebles, Phys. Rev. D 71, 083505 (2005).

[12] R. Bean, E. E. Flanagan, and M. Trodden, Phys. Rev. D 78, 023009 (2008).

[13] M. Kesden and M. Kamionkowski, Phys. Rev. D 74, 083007 (2006).

[14] M. Kesden and M. Kamionkowski, Phys. Rev. Lett. 97, 131303 (2006).

[15] T. Damour, Classical Quantum Gravity 13, A33 (1996).

[16] S. Schlamminger, K. Y. Choi, T.A. Wagner, J.H. Gundlach, and E. G. Adelberger, Phys. Rev. Lett. 100, 041101 (2008).

[17] J. Bovy and G. R. Farrar, Phys. Rev. Lett. 102, 101301 (2009).

[18] S. M. Carroll, S. Mantry, and M. J. Ramsey-Musolf, arXiv:0902.4461.

[19] D. B. Kaplan and M. B. Wise, J. High Energy Phys. 08 (2000) 037.

[20] G. R. Dvali and M. Zaldarriaga, Phys. Rev. Lett. 88, 091303 (2002).

[21] T. Dent, Phys. Rev. Lett. 101, 041102 (2008).

[22] M. A. Shifman, A. I. Vainshtein, and V. I. Zakharov, Phys. Lett. B 78, 443 (1978).

[23] G. Belanger, F. Boudjema, A. Pukhov, and A. Semenov, Comput. Phys. Commun. 180, 747 (2009).

[24] C. W. Stubbs, Phys. Rev. Lett. 70, 119 (1993).

[25] J.P. Blaser, M. Bye, G. Cavallo, T. Damour, C. W.F. Everitt, A. Hedin, R. W. Hellings, Y. Jafry, R. Laurance, and M. Lee, NASA STI/Recon, Technical Report N 95, 26184 (1993).

[26] M. Cirelli, N. Fornengo, and A. Strumia, Nucl. Phys. B753, 178 (2006). 